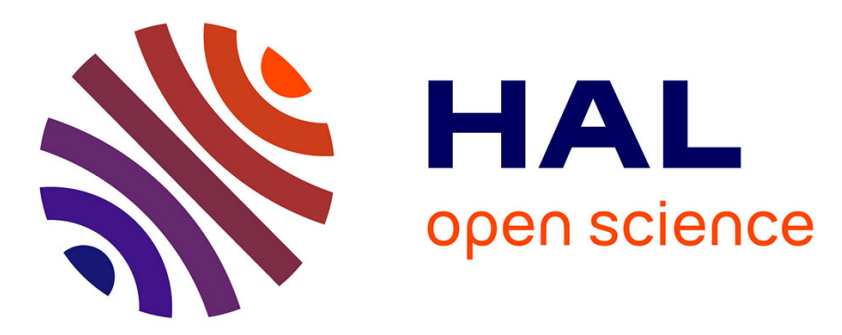

\title{
De novo assembly and functional annotation of the transcriptome of Mimachlamys varia , a bioindicator marine bivalve
}

Amélia Viricel, Vanessa Buren Becquet, Emmanuel Dubillot, Eric Pante

\section{To cite this version:}

Amélia Viricel, Vanessa Buren Becquet, Emmanuel Dubillot, Eric Pante. De novo assembly and functional annotation of the transcriptome of Mimachlamys varia, a bioindicator marine bivalve. Marine Genomics, 2018, 41, pp.42-45. 10.1016/j.margen.2018.04.002 hal-01856136

\author{
HAL Id: hal-01856136 \\ https://hal.science/hal-01856136
}

Submitted on 21 Aug 2018

HAL is a multi-disciplinary open access archive for the deposit and dissemination of scientific research documents, whether they are published or not. The documents may come from teaching and research institutions in France or abroad, or from public or private research centers.
L'archive ouverte pluridisciplinaire HAL, est destinée au dépôt et à la diffusion de documents scientifiques de niveau recherche, publiés ou non, émanant des établissements d'enseignement et de recherche français ou étrangers, des laboratoires publics ou privés. 
1 De novo assembly and functional annotation of the transcriptome

\section{of Mimachlamys varia, a bioindicator marine bivalve}

3

4

5 Amélia Viricel $^{\mathrm{a} *}$, Vanessa Becquet $^{\mathrm{a}}$, Emmanuel Dubillot $^{\mathrm{a}}$, Eric Pante $^{\mathrm{a}}$

6

7 Affiliation :

$8{ }^{a}$ Littoral Environnement et Sociétés (LIENSs), UMR 7266, CNRS-Université de La Rochelle,

92 rue Olympe de Gouges, F-17042 La Rochelle Cedex 01, France.

10

11 Email address of each author :

12

amelia.viricel@gmail.com;

vanessa.becquet@univ-lr.fr;

emanuel.dubillot@univ-lr.fr;

13 eric.pante@univ-lr.fr

14

* Corresponding author: Amélia Viricel

16

Email: amelia.viricel@gmail.com

17

Tel: $+33(0) 546507658$

18

19

20

21

22

23 


\section{Abstract}

25 Developing genomic resources for species used as bioindicators of environmental pollution 26 facilitates identification of new biomarkers of interest. The variegated scallop Mimachlamys 27 varia (Pectinidae) is a marine bivalve used to evaluate and monitor chemical contamination 28 on the French Atlantic coast. Because natural populations of this species are commercially 29 harvested, there is particular interest in understanding its responses to environmental pollution 30 and pathogens. We assembled and annotated the transcriptome of $M$. varia obtained from a 31 pool of five tissue types (gills, mantle, digestive gland, gonad, adductor muscle). In depth 32 Illumina sequencing led to the assembly of 333,022 transcripts, covering $98 \%$ of genes 33 conserved among eukaryotes.

\section{Keywords}

36 Pectinidae, de novo assembly, variegated scallop, functional annotation, RNA-seq, biomarker 37 


\section{Introduction}

Marine bivalves are considered sentinels of environmental quality because they filter large volumes of seawater and bioaccumulate contaminants in high concentrations (Grosell and Walsh 2006). Within this group, the variegated scallop Mimachlamys varia (Pectinidae) has been used in ecotoxicological studies aimed at monitoring environmental contamination levels along the French Atlantic coast (Milinkovitch et al., 2015; Breitwieser et al., 2016 \& 2017). These studies have detected significant physiological responses of this bivalve to chronic chemical pollution through the use of biomarkers linked to oxidative stress, mitochondrial respiration and immune system alteration. Additionally, potential long-term effects of chronic chemical contamination on $M$. varia natural populations have been investigated by comparing genetic diversity among sites along the French Atlantic coast (Breitwieser et al. submitted). Past studies on M. varia have focused on a few target genes for population genetic analyses and genomic resources are still lacking for this bioindicator species. Investigating gene expression would further our understanding of the responses of this bivalve to chemical pollution, and would allow identifying new biomarkers of interest for biomonitoring environmental quality.

Studies investigating transcriptomic responses to chemical contaminant exposure, performed on other marine bivalves, have revealed differential expression of genes implicated in hydrocarbons (e.g. Cai et al. 2014) and heavy metals detoxification (e.g. Meng et al. 2013). Additionally, several pectinids such as M. varia and Pecten maximus are harvested for human consumption, and there has also been a growing interest in developing genomic resources to study bivalve immune responses to pathogens (e.g. Pauletto et al. 2014, Gómez-Chiarri et al. 2015). The transcriptomes of other pectinids have been recently described (e.g. Chlamys farreri: Cai et al. 2014; Chlamys nobilis: Liu et al. 2015). The reference transcriptome of 
Mimachlamys varia will i) facilitate upcoming studies of differential gene expression analyses on this bioindicator species, and ii) provide a valuable genomic resource for future comparative transcriptomic studies of pectinid bivalves.

\section{Data description}

Sampling, RNA extraction and Illumina sequencing

An adult male variegated scallop (shell length: $46 \mathrm{~mm}$, shell height: $40 \mathrm{~mm}$ ) was collected in the sublittoral zone of Angoulins, France (Table 1) at low tide in October 2016. Total RNA was isolated from five distinct tissues collected from this individual (digestive gland, mantle, gills, adductor muscle and gonads), using $50 \mathrm{mg}$ of each tissue type. RNA extractions were performed using the Nucleospin RNA Set for Nucleozol kit (Macherey-Nagel). After determining RNA concentration using a Nanodrop 2000 spectrophotometer (Thermo Scientific), the five RNA extractions were pooled in equal amounts ( $4 \mu \mathrm{g}$ of RNA per tissue type). The quality of the RNA pool was assessed on an Agilent Bioanalyzer before poly(A) enrichment and normalized random primed cDNA library preparation. The library was sequenced using an Illumina HiSeq 2500 with a modified protocol producing long paired-end reads $(2 \times 300 \mathrm{bp})$. Sample and sequencing information is given in Table 1 following MIxS standard descriptors (Yilmaz et al. 2011).

\begin{tabular}{ll}
\hline Item & Description \\
\hline Investigation_type & Eukaryote \\
Project_name & Reference transcriptome for Mimachlamys varia \\
Lat_Lon & $46.09880 ;-1.12740$ \\
Geo_loc_name & Bay of Biscay, France \\
Collection_date & $17-$ October-2016 \\
Environment & Marine water \\
Biome & ENVO:01000410 \\
Feature & ENVO:01000105 \\
env_Material & ENVO:00002150
\end{tabular}


Sequencing method

Assembly method

Accession number of raw reads

Accession number of transcripts
Illumina HiSeq 2500 paired-end 2x300 bp

Trinity v 2.4 .0

SRP127478

GGGO00000000
85

Table 1. Data description following MIxS standards.

\section{De novo transcriptome assembly and quality control}

Sequencing produced 64,291,972 raw reads that were trimmed and quality filtered using Trimmomatic v. 0.36 (parameters : HEADCROP:10 LEADING:15 TRAILING:15

SLIDINGWINDOW:4:15 MINLEN:100) including adapter removal (ILLUMINACLIP: 2:30:10) (Table 2). Reads were then filtered using Deconseq v. 0.4.3 to remove potential transcripts from human, bacteria, viruses, archae and microalgae that could be present in scallop tissues as environmental or laboratory contaminants. In addition to the Deconseq transcript databases, we included the SILVA (complete database release 128, Quast et al. 2013), MarREF and MarDB (Klemetsen et al. 2017) databases, and 4 microalgae genomes (Accession nb NW_011934117.1, NW_005202428.1, NC_011669.1 and NC_012064.1) in this decontamination step. SILVA is a comprehensive database of ribosomal RNA (rRNA) sequences (including Bacteria, Archaea and Eukarya), and thus allowed removal of potentially remaining endogenous and microbial rRNAs from our transcriptome data. The MarREF and MarDB databases comprise genome sequences from marine prokaryotes that could have been present in our sample, particularly in the gut content. In total, 178,425 reads (0.29\% of all quality-filtered reads) were excluded using Deconseq (search parameters: $90 \%$ minimum identity, 50\% minimum coverage). Read quality was assessed using FastQC for raw reads and after each quality control step (Trimmomatic and Deconseq).

The de novo assembly was achieved using Trinity v.2.4.0 (Grabherr et al. 2011) with default parameters and in-silico normalization. Transdecoder was used to identify putative coding 
108 regions within transcripts (ORFs $\geq 100$ AA long, homology to known proteins determined 109 using blastp and pfam searches following http://transdecoder.github.io). Four metrics were

110 used to assess assembly quality, following recommendations from Honaas et al. (2016). First,

111 the proportion of quality-filtered reads mapping back to the assembly was high (93.3\%).

112 Second, the N50 based on the longest isoform per gene was 1,378 bp. Third, $98.0 \%$ and

$11397.9 \%$ genes that are conserved and widely expressed in eukaryotes and metazoans,

114 respectively, were recovered in our Transdecoder candidate ORFs, as determined using

115 BUSCO v. 3 (Simão et al 2015 ; Waterhouse et al 2017). Finally, the total number of

116 transcripts $(n=333,022)$ and genes (sensu Trinity; $n=180,900)$ is consistent with other

117 pectinid bivalve assemblies (e.g. Mizuhopecten yessoensis: Meng et al. 2013; Chlamys

118 nobilis: Liu et al. 2015). Other assembly statistics are described in Table 2.

119 Prior to annotation, Transdecoder candidate ORFs were blasted (blastp, evalue 1e-5 cutoff)

120 against a custom database of 369,629 sequences of non-eukaryotes (viruses, bacteria, archaea)

121 assembled from the curated UniProtKB/Swiss-Prot database (2018-01-30). All sequences

122 returning a significant match to these taxa were excluded.

\section{Transcriptome annotation}

125 Transcript annotation was performed using Blast2GO PRO v. 5.0 .8 based on 78,784

126 Transdecoder candidate ORFs (Supplementary files 1, 2 and 3). Blast searches were

127 performed using blastp (Altschul et al. 1997) against the UniProtKB/ Swiss-Prot database

128 (2017-06-06), using an e-value threshold of 1e-3 and retaining 20 blast hits per query. We

129 chose to use a rather lax e-value since we were comparing data from a non-model organism to

130 the curated database. Mapping and annotation were performed in Blast2GO PRO v. 5.0.8 with

131 default settings. The InterProScan pipeline (Finn et al. 2017) was run and GO terms were

132 merged to the Blast2GO annotation. ANNEX annotation augmentation was performed 
133 (Myhre et al 2006). A total of 36,278 peptide sequences (46\%) were annotated (Figure 1).

134 Among those, we detected enzymes involved in immune response (phenol oxidases such as

135 genes belonging to the Laccase family), oxidative stress response (e.g. glutathione

136 peroxidase) and toxin biotransformation (e.g. glutathine S-transferase), which are commonly

137 used as biomarkers in ecotoxicological studies on invertebrates (e.g. Valavanidis et al. 2006;

138 Breitwieser et al. 2016; Luna-Acosta et al. 2017).

139

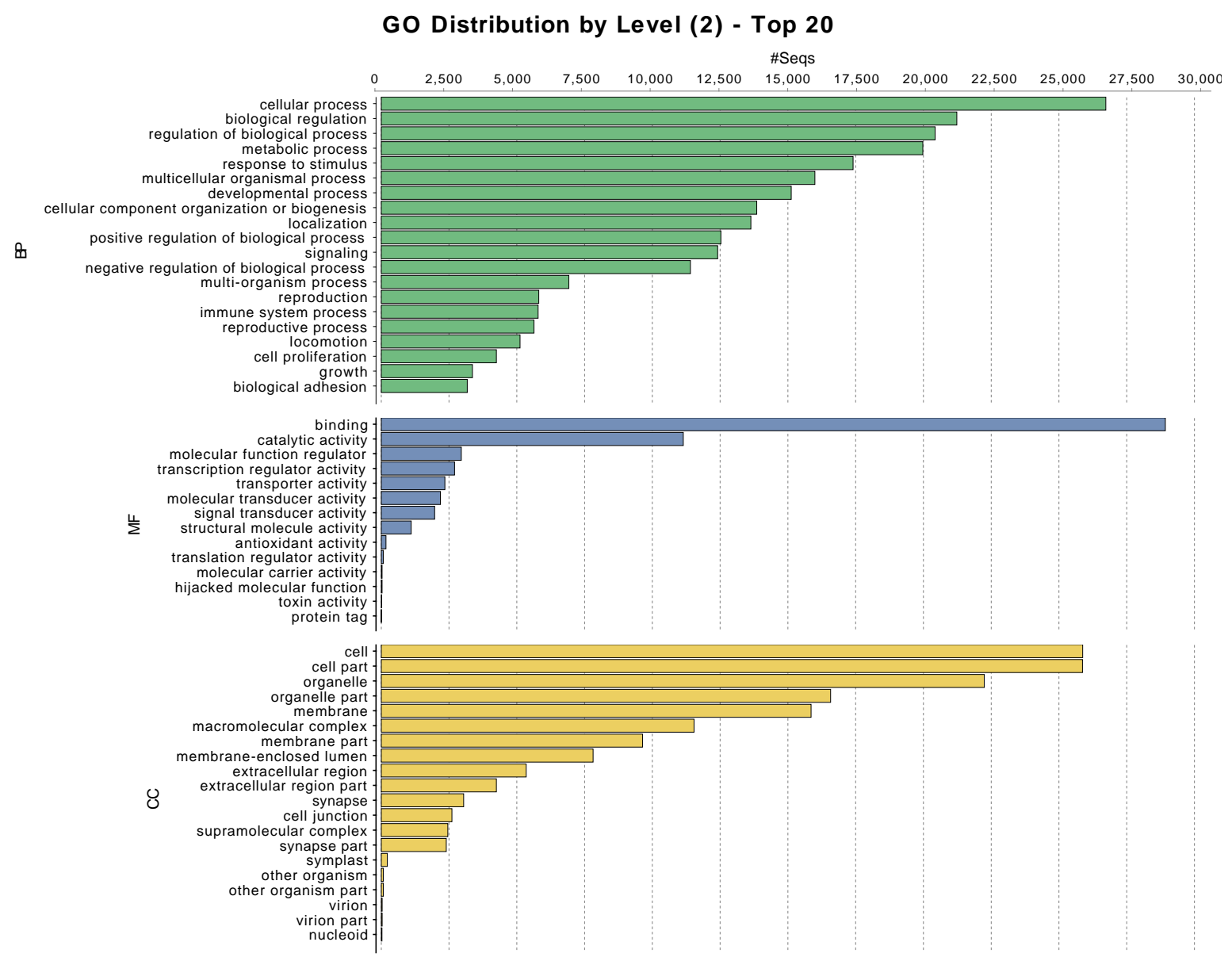

140

141 Figure 1. Gene ontology (GO) functional categories.

142

143 Matches to other Pteriomorphia bivalves

$14479.5 \%$ of the 78,784 Transdecoder candidate ORFs blasted to a custom Pteriomorphia (a

145 subclass of Bivalvia including scallops, oysters and mussels) TrEMBL database (2018-02- 
146 06). Among these, $79.5 \%$ of peptides best hits corresponded to the Yesso scallop

147 (Mizuhopecten yessoensis), which reference genome was recently sequenced (Wang et al

148 2017). Best hits to Crassostrea gigas represented 9.9\%. Other best hits (including Pectinidae,

149 Ostreidae, Mytilidae, Pteriidae and Arcidae) represented $0.2 \%$ of peptide sequences.

150

151

152

\begin{tabular}{ll}
\hline Raw reads & $64,291,972$ \\
Quality-filtered reads (prior to Deconseq) & $62,039,219$ \\
Total assembled bases & $148,351,501$ \\
\% reads mapping back to assembly & 93.3 \\
Number of transcripts & 333,022 \\
Number of genes & 180,900 \\
Median contig length (bp) & 445 \\
Average contig length (bp) & 820 \\
contig N50 (in bp, based on the longest isoform) & 1,378 \\
Transdecoder peptides & 78,784 \\
BUSCO Eukaryote & C: $98.0 \%$ [S: $43.9 \%$, D: $54.1 \%]$ \\
& F: $1.3 \%$, M: $0.7 \%$, n: 303 \\
BUSCO Metazoa & C: $97.9 \%$ [S: $41.9 \%$, D: $56.0 \%]$, \\
& F: $1.0 \%$, M: $1.1 \%$, n: 978 \\
\hline
\end{tabular}

153

154 Table 2. Assembly statistics. These statistics are based on the longest isoform per gene. The

155 terminology corresponds to assembly with Trinity. BUSCO codes indicate the percentage of

156 widely expressed genes that were recovered completely (C) (for single-copy (S) and

157 duplicated (D) genes), that were only partially recovered (F for "Fragmented"), or that were

158 missing (M). The total number of orthologous groups of genes (n) that was searched in

159 BUSCO is also indicated.

160

161

Data availability 
162 Raw reads are available through the NBCI Sequence Read Archive (SRP127478). The

163 Transcriptome Shotgun Assembly project has been deposited at DDBJ/EMBL/GenBank

164 under the accession GGGO00000000. The version described in this paper is the first version,

165 GGGO01000000. Both data sources are linked to the NCBI BioSample and BioProject

166 numbers SAMN08235964 and PRJNA427371, respectively.

167

168 Acknowledgments

169 This work was supported by the contrat de plan Etat-Région (CPER/FEDER) ECONAT

170 (RPC DYPOMAR). We would like to thank Nathalie Imbert and Denis Fichet for

171 coordinating axe 2 of DYPOMAR and Benoit Simon-Bouhet for additional funding. RNA

172 extractions were prepared at the Molecular Core Facility at the University of La Rochelle. We

173 thank two anonymous reviewers for their constructive comments on the manuscript.

174

175 Supplementary data

176 Supplementary data to this article can be found at $\mathrm{xxxx}$.

177

178 References

179 Altschul, S.F., Madden, T.L., Schäffer, A.A., Zheng Zhang J.Z., Miller, W. and Lipman, D.J.

180 1997. Gapped BLAST and PSI-BLAST: a new generation of protein database search

181 programs. Nucleic Acids Research 25:3389-3402.

182 Bolger, A.M., Lohse, M., Usadel, B. 2014. Trimmomatic: a flexible trimmer for Illumina

183 sequence data. Bioinformatics 30, 2114-2120

184 Breitwieser, M., Viricel, A., Graber, M., Murillo, L., Becquet, V., Churlaud, C., Fruitier-

185 Arnaudin, I., Huet, V., Lacroix, C., Pante, E., Le Floch, S., Thomas-Guyon, H. 2016. 
Short-term and long-term biological effects of chronic chemical contamination on natural populations of a marine bivalve. PLoS ONE 11, e0150184

Breitwieser, M., Viricel, A., Churlaud, C., Guillot, B., Martin, E., Stenger, P-L., Huet, V., Fontanaud, A., Thomas-Guyon, H. 2017. First data on three bivalve species exposed to an intra-harbour polymetallic contamination (La Rochelle, France). Comparative Biochemistry and Physiology, Part C 199, 28-37

Breitwieser, M., Becquet, V., Thomas-Guyon, H., Pillet, V., Sauriau, P-G., Graber, M., Viricel, A. (submitted) Genetic evidence for a single population of variegated scallop Mimachlamys varia across a biogeographic break on the French coastline. Submitted to Journal of Molluscan Studies

Cai, Y., Pan, L., Hu, F., Jin, Q., Liu, T. 2014. Deep sequencing-based transcriptome profiling analysis of Chlamys farreri exposed to benzo[a]pyrene. Gene 551,261-270 Dosztányi, Z., El-Gebali, S., Fraser, M., Gough, J., Haft, D., Holliday, G.L., Huang, H., Huang, X., Letunic, I., Lopez, R., Lu, S., Marchler-Bauer, A., Mi, H., Mistry, J., Natale, D.A., Necci, M., Nuka, G., Orengo, C.A., Park, Y., Pesseat, S., Piovesan, D., Potter, S.C., Rawlings, N.D., Redaschi, N., Richardson, L., Rivoire, C., Sangrador-Vegas, A., Sigrist,

\section{D199}

Gómez-Chiarri, M., Guo, X., Tanguy, A., He, Y., Proestou, D. 2015. The use of -omic tools

210 Grabherr, M.G., Haas, B.J., Yassour M., Levin, J.Z., Thompson, D.A., Amit, I., Adiconis, X., 211 Fan, L., Rauchowdhury, R., Zeng, Q., Chen, Z., Mauceli, E., Hacohen, N., Gnirke, A., 
Rhind, N., di Palma, F., Birren, B.W., Nusbaum, C., Lindblad-Toh, K., Friedman, N., Regev, A. 2011. Full-length transcriptome assembly from RNA-se data without a reference genome. Nat Biotechnol. 29, 644-652

Grosell, M., Walsh, P.J. 2006. Sentinel species and animal models of human health.

Honaas, L.A., Wafula, E.K., Wickett, N.J., Der, J.P., Zhang, Y., Edger, P.P., Altman, N.S., 218 Pires, J.C., Leebens-Mack, J.H., dePamphilis C.W. 2016. Selecting superior de novo transcriptome assemblies : lessons learned by leveraging the best plant genome. PLoS ONE 11(1), e0146062

Klemetsen, T., Raknes, I.A., Fu, J., Agafonov, A., Balasundaram, S.V., Tartari, G., Robertsen, E., Willassen, N.P. 2017. The MAR databases: development and implementation of databases specific for marine metagenomics. Nucl. Acids. Res, gkx1036 focusing on mining transcripts for carotenoid-based coloration. BMC Genomics 16:44 phenoloxidases in bivalves. Marine Pollution Bulletin 122, 5-16

Meng, X-L., Liu, M., Jiang, K-y., Wang, B-j., Tian, X., Sun, S-j., Luo, Z-y., Qiu, C-w., Wang, L. 2013. De novo characterization of Japanese scallop Mizuhopecten yessoensis transcriptome and analysis of its gene expression following cadmium exposure. PLoS ONE, 8, e64485

234 2015. In situ evaluation of oxidative stress and immunological parameters as ecotoxicological biomarkers in a novel sentinel species (Mimachlamys varia). Aquatic Toxicology 161, 170-175 
Myhre, S., Tveit, H., Mollestad, T., Laegreid, A. 2006. Additional gene ontology structure for improved biological reasoning. Bioinformatics 22, 2020-7

Pauletto, M., Milan, M., Moreira, R., Novoa, B., Figueras, A., Babbucci, M., Patarnello, T., Bargelloni, L. 2014. Deep transcriptome sequencing of Pecten maximus hemocytes : A genomic resource for bivalve immunology. Fish \& Shellfish Immunology 37, 154-165

Quast, C., Pruesse, E., Yilmaz, P., Gerken, J., Schweer, T., Yarza, P., Peplies, J., Glöckner, F.O. 2013. The SILVA ribosomal RNA gene database project: improved data processing and web-based tools. Nucl. Acids. Res 41(D1), D590-D596

Simão, F.A., Waterhouse, R.M., Ioannidis, P., Kriventseva, E.V., Zdobnov, E.M. 2015. BUSCO: assessing genome assembly and annotation completeness with single-copy orthologs. Bioinformatics 31, 3210-3212

Valavanidis, A., Vlahogianni, T., Dassenakis, M., Scoullos, M. 2006. Molecular biomarkers of oxidative stress in aquatic organisms in relation to toxic environmental pollutants. Ecotoxicology and Environmental Safety 64, 178-189

Wang, S., Zhang, J., Jiao, W., Li, J., Xun, X., Sun, Y., Guo, X., Huan, P., Dong, B., Zhang, L., et al. 2017. Scallop genome provides insights into evolution of bilaterian karyotype and development. Nature Ecology \& Evolution 1, 0120.

Waterhouse, R.M., Seppey, M., Simão, F.A., Manni, M., Ioannidis, P., Klioutchnikov, G., Kriventseva, E.V., Zdobnov, E.M. 2017. BUSCO applications from quality assessments to gene prediction and phylogenomics. Molecular Biology and Evolution, doi:

$$
10.1093 / \mathrm{molbev} / \mathrm{msx} 319
$$

Yilmaz, P., Kottmann, R., Field, D., Knight, R., Cole, J.R., Amaral-Zettler, L., Gilbert, J.A., Karsch-Mizrachi, I., Johnston, A., Cochrane, G., et al. 2011. Minimum information about a marker gene sequence (mimarks) and minimum information about any (x) sequence (mixs) specifications. Nature Biotechnology 29, 415-420. 